\title{
Remote coronal dimmings related to a circular-ribbon flare ${ }^{\star}$
}

\author{
Q. M. Zhang ${ }^{1,2,3}$ and R. S. Zheng ${ }^{4}$
}

\author{
1 Key Laboratory of Dark Matter and Space Science, Purple Mountain Observatory, CAS, Nanjing 210033, PR China \\ e-mail: zhangqm@pmo.ac.cn \\ 2 State Key Laboratory of Lunar and Planetary Sciences, Macau University of Science and Technology, Macau, PR China \\ 3 State Key Laboratory of Space Weather, Chinese Academy of Sciences, Beijing 100190, PR China \\ ${ }^{4}$ Shandong Key Laboratory of Optical Astronomy and Solar-Terrestrial Environment, School of Space Science and Physics, Institute \\ of Space Sciences, Shandong University, Weihai, Shandong 264209, PR China \\ e-mail: ruishengzheng@sdu.edu.cn
}

Received 17 November 2019 / Accepted 19 December 2019

\begin{abstract}
Aims. In this paper, we report multiwavelength observations of remote coronal dimmings related to an M1.1 circular-ribbon flare in active region (AR) 12434 .

Methods. The confined flare without a coronal mass ejection was observed by the Atmospheric Imaging Assembly (AIA) and the Helioseismic and Magnetic Imager on board the Solar Dynamics Observatory on 2015 October 16. We obtained global three-dimensional (3D) magnetic fields before the flare using the potential field source surface modeling.

Results. A few minutes before the flare hard X-ray peak time (06:13:48 UT), small-scale, weak dimming appeared $\sim 240^{\prime \prime}$ away from the flare site, which can be observed by AIA only in 131 and $171 \AA$ A. Afterward, long and narrow dimmings became evident in all AIA extreme-ultraviolet passbands except $304 \AA$, while localized core dimming was not clearly observed near the flare site. The large-area dimmings extended southeastward and the areas increased gradually. The total area of dimmings reaches $(1.2 \pm 0.4) \times 10^{4} \mathrm{Mm}^{2}$ in $193 \AA$. The maximal relative intensity decreases in 171 and $193 \AA$ reach $90 \%$ and $80 \%$, respectively. Subsequently, the dimmings began to replenish and the area decreased slowly, lasting for $\geq 3 \mathrm{~h}$. The remote dimmings and AR 12434 are connected by large-scale coronal loops. The remote dimmings are associated with the southwest footpoints of coronal loops with weak negative polarities. Possible origins of remote dimmings are discussed.
\end{abstract}

Key words. Sun: corona - Sun: flares - Sun: filaments, prominences - Sun: magnetic fields

\section{Introduction}

Coronal dimmings are transient regions of greatly reduced emission in extreme-ultraviolet (EUV) and soft X-ray (SXR) wavelengths (Hudson et al. 1996; Thompson et al. 1998, 2000). They are tightly related to coronal mass ejections (CMEs) and solar flares (Reinard \& Biesecker 2009; Krista \& Reinard 2017; Dissauer et al. 2018a,b, 2019; Veronig et al. 2019). When a CME rises and erupts into interplanetary space, the coronal source region is impulsively evacuated and the plasma density is heavily depleted (Harrison \& Lyons 2000; Harrison et al. 2003; Mason et al. 2014). The percentage of density depletion could reach up to 50-70\% (Vanninathan et al. 2018). Persistent upflows at speeds of hundreds of $\mathrm{km} \mathrm{s}^{-1}$ have been observed using the spectroscopic observations (Harra \& Sterling 2001; Harra et al. 2007; Attrill et al. 2010; Dolla \& Zhukov 2011; Tian et al. 2012; Veronig et al. 2019). The Doppler velocities depend on the formation temperatures of ions (Jin et al. 2009), and the most significant dimmings can be observed at temperatures of a few million Kelvin (MK; Zhukov \& Auchère 2004; Robbrecht \& Wang 2010).

According to the location, coronal dimmings can be classified into deep core dimmings near the flare site and widespread diffuse dimmings (Zarro et al. 1999; Mandrini et al. 2005; Attrill et al. 2007; Delannée et al. 2007). Deep core dimmings

\footnotetext{
* Movies associated to Figs. 1, 3, 4, and 6 are available at https://www . aanda.org
}

or twin dimmings are related to the footpoints of a flux rope, while secondary diffuse dimmings result from continuing field line stretching during the eruption (Mandrini et al. 2007; Miklenic et al. 2011; Dissauer et al. 2018b; Vanninathan et al. 2018). The widths of dimmings are positively correlated with the CME widths (Reinard \& Biesecker 2008). The velocity and mass of CMEs are found to be intimately related to the slope and depth of the dark voids left behind CMEs (Mason et al. 2016).

In most cases, coronal dimmings appear in the impulsive phase of a flare that is consistent with the acceleration phase of the corresponding CME (Cheng \& Qiu 2016). The typical evolution of a dimming area is characterized by a sharp rise followed by a slow recovery via magnetic reconnection (Attrill et al. 2006; Reinard \& Biesecker 2008). The total area and unsigned magnetic flux of dimmings are $\sim 10^{10} \mathrm{~km}^{2}$ and $\sim 10^{21} \mathrm{Mx}$ (Dissauer et al. 2018b). The total lifetime of dimmings ranges from $3 \mathrm{~h}$ to $12 \mathrm{~h}$ with an average value of $\sim 8 \mathrm{~h}$ (Reinard \& Biesecker 2008). Using the high-resolution and multiwavelength observations from the Atmospheric Imaging Assembly (AIA; Lemen et al. 2012) on board the Solar Dynamics Observatory (SDO), Zhang et al. (2017) discovered pre-flare coronal dimmings, which lasted for $\sim 96 \mathrm{~min}$ before the impulsive phase of an eruptive flare. The authors proposed that preflare dimming serves as a precursor of twin dimmings. Additional events about pre-eruption dimmings were investigated and reported (Qiu \& Cheng 2017; Wang et al. 2019).

During the whole evolution of NOAA active region (AR) 12434 in 2015 October, a series of homologous and 


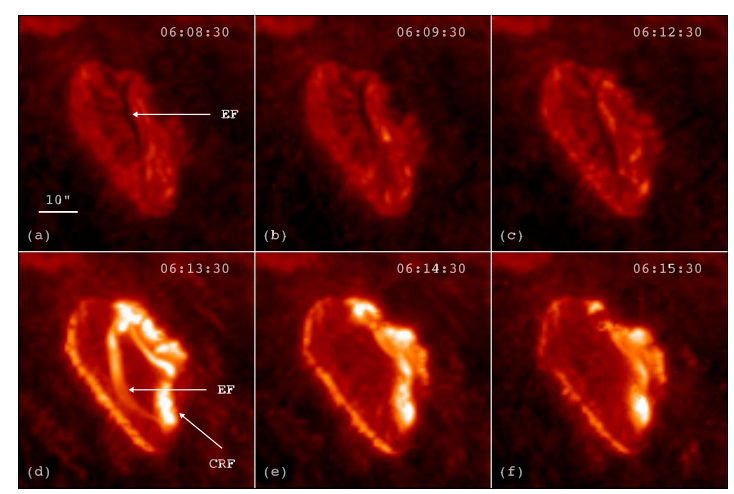

Fig. 1. Six snapshots of the original AIA $304 \AA$ images. The white arrows point to the EF that triggered the CRF. The field of view is $60^{\prime \prime} \times 60^{\prime \prime}$. The evolution of EF is shown in a movie (anim0.mov) available online.

short-lived circular-ribbon flares (CRFs) were produced (Zhang et al. 2016a,b). Zhang et al. (2019) calculated the energy partition of two M1.1 CRFs taking place on October 15 and 16 , respectively. In this paper, remote coronal dimmings related to the M1.1 flare on October 16 are investigated to explore the relationship between CRFs and coronal dimmings. Observations and data analysis are described in Sect. 2. Discussions and a brief summary are shown in Sect. 3 .

\section{Observations and data analysis}

The M1.1 flare was observed by AIA (Zhang et al. 2019). The AIA takes full-disk images in seven EUV wavelengths (94, 131, $171,193,211,304$, and $335 \AA$ ) with a cadence of $12 \mathrm{~s}$ and in two UV wavelengths (1600 and $1700 \AA$ ) with a cadence of $24 \mathrm{~s}$. The photospheric line-of-sight (LOS) magnetograms are observed by the Helioseismic and Magnetic Imager (HMI; Scherrer et al. 2012) on board SDO with a cadence of $45 \mathrm{~s}$. The spatial resolutions of AIA and HMI are 1 '!2.

Figure 1 shows six snapshots of the AIA $304 \AA$ images. It is clear that the CRF was triggered by the eruption of a minifilament at $\sim 06: 13$ UT. During its eruption, the arc-shaped eruptive filament (EF) was heated and became bright in $304 \AA$. The SXR light curves of the flare with a cadence of $\sim 2.05 \mathrm{~s}$ are plotted in Fig. 2a. The flux in $1-8 \AA$ increased rapidly from $\sim 06: 11$ UT to $\sim 06: 16 \mathrm{UT}$, which was followed by a gradual decay phase until $\sim 06: 35$ UT. The hard X-ray (HXR) flux at 25-50 keV observed by the Reuven Ramaty High-Energy Solar Spectroscopic Imager (RHESSI; Lin et al. 2002) is plotted in Fig. 2b. The peak time (06:13:48 UT) indicative of the highest energy release rate is denoted by the black dashed line.

The confined flare was neither associated with a CME nor with localized core dimming near the flare site. Instead, it was related to remote dimmings $\sim 240^{\prime \prime}$ away in the quiet region. To better illustrate the coronal dimmings, the base-ratio technique is applied by taking the EUV images at 06:00:00 UT as base images (Dissauer et al. 2018a). Figure 3 shows four snapshots of base-ratio images during 06:07-06:11 UT in $131 \AA$ and $171 \AA$. It is surprising that small-scale, narrow dimming appeared to the southwest of flare a few minutes before the onset of flare, which is considered as pre-flare dimming. The area increased very slowly with the intensity decreasing. We checked the baseratio images in other EUV wavelengths and found that pre-flare dimming was prominent only in 131 and $171 \AA$ with formation temperatures of $\log T \approx 5.6-5.8$ in the lower corona.

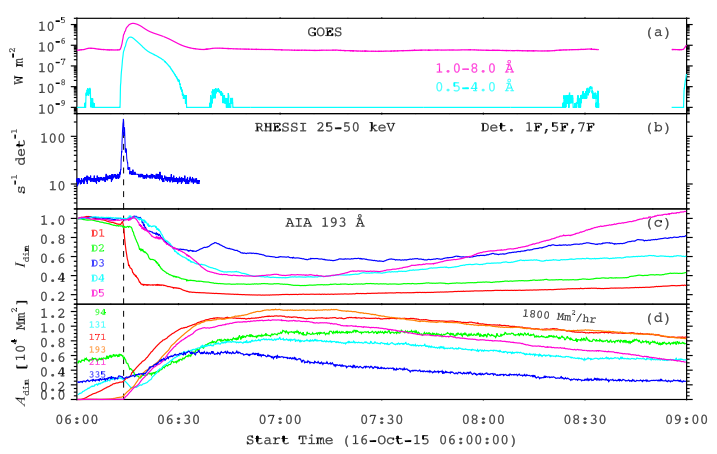

Fig. 2. Panel $a$ : GOES light curves of the flare. Panel $b$ : HXR light curve at $25-50 \mathrm{keV}$. Panel $c$ : evolutions of the average intensities of "D1""D5" in Fig. 4e. Panel $d$ : time evolutions of the total area of remote dimmings in different wavelengths. The black dashed line denotes the time at 06:13:48 UT.

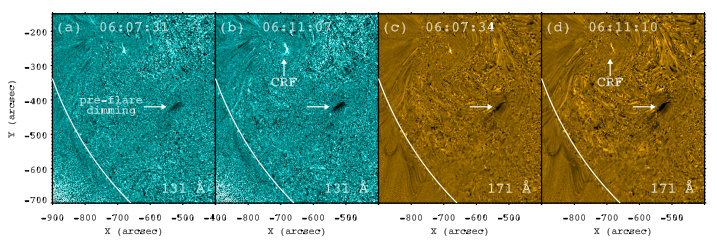

Fig. 3. Base-ratio images from AIA in 131 and $171 \AA$. The horizontal and vertical arrows point to the pre-flare dimming (dark area) and CRF. The evolution of pre-flare dimming is shown in a movie (anim1.mov) available online.

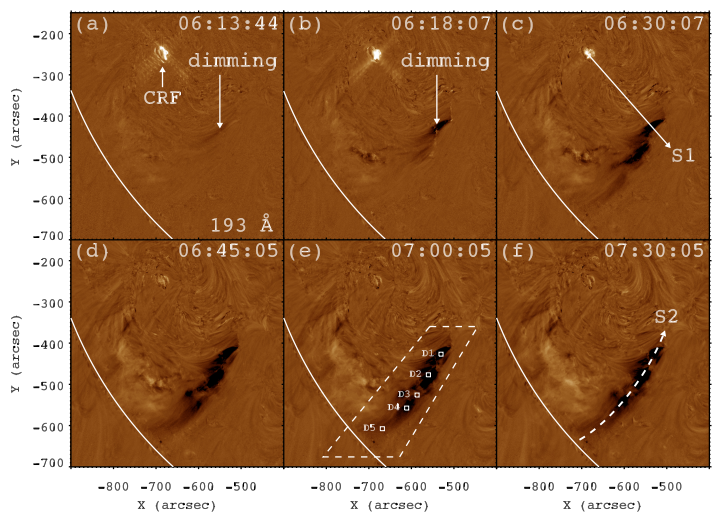

Fig. 4. Base-ratio images from AIA in $193 \AA$ showing the remote coronal dimmings (dark area). The evolution of large-area dimmings is shown in a movie (anim2.mov) available online.

In Fig. 4, the $193 \AA$ base-ratio images illustrate the evolution of large-area remote dimmings after the onset of flare. Long and narrow dimmings, which were cospatial with the pre-flare dimming, appeared shortly after $\sim 06: 14$ UT. As time went on, the dimmings extended southeastward with the area increasing rapidly. The dimmings were replenished and the area reduced slowly after the maxima. The temporal and spatial relationships between pre-flare dimming and large-area dimmings suggest that remote dimmings started from the pre-flare dimming and developed into large-area dimmings. Hence, the pre-flare dimming may serve as a precursor of large-area dimmings.

In Fig. 4e, five small boxes ("D1"-“D5") within the dimmings are selected and investigated. Time evolutions of the average base-ratio intensities within the five boxes in $193 \AA$ are plotted in Fig. 2c. The intensity evolution is characterized by a sharp decrease followed by a gradual recovery. It is obvious that 
Table 1. Maximal area $\left(\times 10^{4} \mathrm{Mm}^{2}\right)$ and relative intensity decrease (\%) of the remote dimmings in multiple wavelengths.

\begin{tabular}{lccc}
\hline \hline$\lambda(\AA)$ & 94 & 131 & 171 \\
\hline Area & $0.67-1.28$ & $0.4-1.42$ & $0.78-1.70$ \\
Percent & $15-70$ & $20-75$ & $55-92$ \\
\hline$\lambda(\AA)$ & 193 & 211 & 335 \\
\hline Area & $0.84-1.79$ & $0.70-1.78$ & $0.28-1.29$ \\
Percent & $45-80$ & $30-55$ & $10-55$ \\
\hline
\end{tabular}

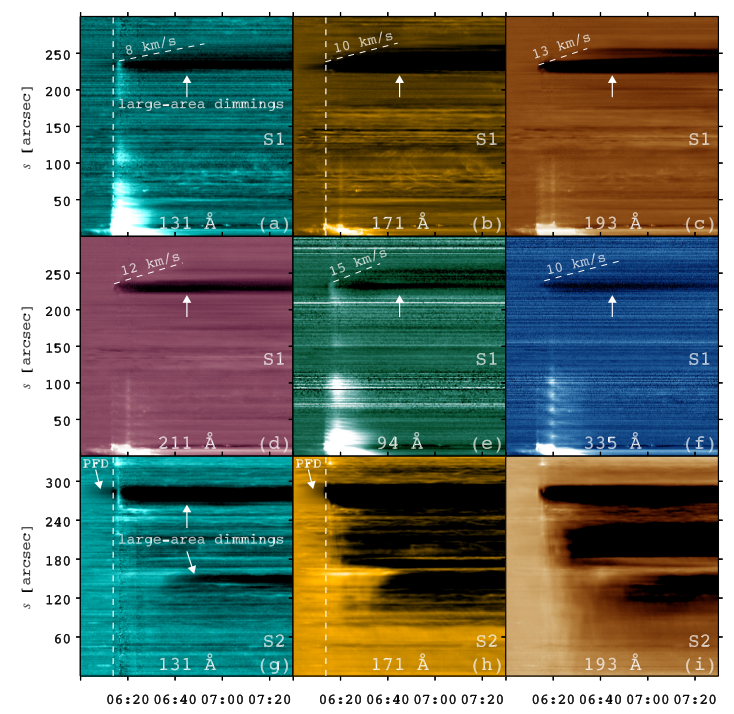

Fig. 5. Panels $a-f$ : time-distance diagrams of S1. In panels $a$ and $b$, the white dashed lines denote the HXR peak time (06:13:48 UT). Panels $g-i$ : time-distance diagrams of S2.

the intensity depletion of D1 preceded that of D2. The depletions of D3-D5 were further delayed, which is consistent with the southeastward extension of large-area remote dimmings. Moreover, the relative intensity decrease of D1 reaches $\sim 80 \%$, which is considerably higher than that of D3 $(\sim 45 \%)$. Owing to the different formation temperatures and heights of AIA EUV passbands, the degrees of intensity depletion are different accordingly. In Table 1, the maximal relative intensity decreases of the large-area dimmings are listed. It is revealed that the dimmings can remarkably be detected in 171,193 , and $211 \AA$, and are less remarkable in 94, 131, and $335 \AA$ (Vanninathan et al. 2018).

In Fig. $4 \mathrm{e}$, the remote dimmings are encompassed by white dashed lines. The total area of dimmings is defined as the sum of pixels whose intensities are less than $85 \%$ of the pre-flare level at 06:00 UT. Time evolutions of the dimming area in different wavelengths are plotted in Fig. 2d. It is found that the area increased quickly to the apex around 07:16 UT before declining tardily. The lifetime of dimmings exceeded $3 \mathrm{~h}$, with a rise time of $\sim 1 \mathrm{~h}$ and a recovery time of $\geq 2 \mathrm{~h}$. The recovery rate $\left(\sim 1800 \mathrm{Mm}^{2} \mathrm{~h}^{-1}\right)$ of large-area dimmings is labeled in Fig. $2 \mathrm{~d}$. Assuming a constant recovery rate, the dimmings would be totally recovered $\sim 4.8 \mathrm{~h}$ after 09:00 UT. Therefore, an upper limit of the lifetime of dimmings is nearly $8 \mathrm{~h}$. The dimming area reaches $\sim 1.2 \times 10^{4} \mathrm{Mm}^{2}$ in $193 \AA$ and amounts to only $6000-8000 \mathrm{Mm}^{2}$ in 131 and $335 \AA$. In Table 1, the maximal total areas of the remote dimmings in different wavelengths are listed when the intensity threshold changes from $75 \%$ to $95 \%$ of the pre-flare levels.

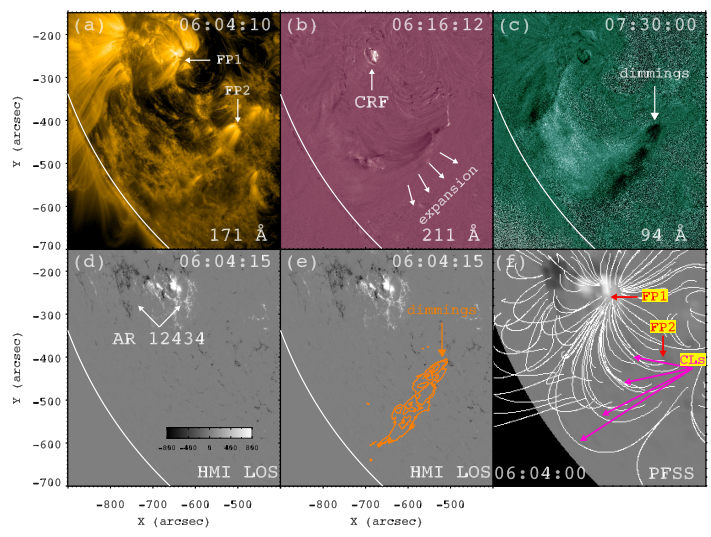

Fig. 6. Panel $a$ : original AIA $171 \AA$ A image. The white arrows point to the footpoints of the large-scale, overlying CLs. Panels $b, c$ : base-ratio images in $211 \AA$ and $94 \AA$. Panels d,e: LOS magnetogram, which is superposed by intensity contours of $193 \AA$ image in Fig. 4e. Panel $f$ : global magnetic fields obtained from the PFSS modeling. The evolution of remote coronal dimmings in $211 \AA$ and $94 \AA$ is shown in a movie (anim3.mov) available online.

In Fig. 4c, an artificial slice (S1) with a length of $300^{\prime \prime}$ is selected, which starts from the flare core and goes across the large-area dimmings. Time-distance diagrams of S1 in various wavelengths are shown in Fig. 5a-f. It is obvious that localized core dimming near the flare was not observed. As soon as the flare occurred, remote dimmings appeared about $240^{\prime \prime}$ away. The apparent speeds of expansion of dimmings along S1 are measured to be $8-15 \mathrm{~km} \mathrm{~s}^{-1}$, with a mean value of $\sim 11 \mathrm{~km} \mathrm{~s}^{-1}$. In Fig. 4f, a curved slice (S2) with a length of $338^{\prime \prime} .5$ is selected, which goes through the entire remote dimmings. Time-distance diagrams of $\mathrm{S} 2$ in various wavelengths are shown in Fig. $5 \mathrm{~g}-\mathrm{i}$, indicating that the extraordinary large-area dimmings extended in the southeast direction.

In Fig. 6, the original AIA image in $171 \AA$ before flare is shown in panel a. Two arrows point to the footpoints (FP1 and FP2) of imaginary large-scale coronal loops (CLs), which are more distinct in $211 \AA$. In panel b, the base-ratio image in $211 \AA$ at 06:16:12 UT is shown. The oblique arrows indicate the directions of quick expansion of the CLs. The apparent speeds of southwestward expansion are indicated in Fig. 5. In Fig. 6e, the LOS magnetogram at 06:04:15 UT is superposed by intensity contours of $193 \AA$ image in Fig. 4e. In AR 12434, FP1 is associated with strong positive polarity, while FP2 in the quiet region is associated with weak negative polarities. In Fig. $6 f$, the global magnetic fields obtained from the potential field source surface (PFSS; Schrijver \& De Rosa 2003) modeling at 06:04:00 UT is depicted, where a series of closed magnetic loops are indicated by magenta arrows. Figure $6 \mathrm{c}$ shows the base-ratio image in $94 \AA$ at 07:30:00 UT, where the bright patch corresponds to largescale CLs and the dark dimmings correspond to the footpoints (FP2) of CLs. Combining the EUV observations, LOS magnetogram, and 3D magnetic configuration, we conclude that FP1 and FP2 are the footpoints of large-scale, overlying CLs.

\section{Discussion and summary}

As mentioned in Sect. 1, there are two types of coronal dimmings in EUV wavelengths: core dimming and widespread diffuse dimmings, which cover a large fraction of the solar surface (e.g., Thompson et al. 1998; Delannée et al. 2007; Dissauer et al. 2018b). Dimmings are mainly due to sudden density depletion 
when CMEs or flares take place (Harrison \& Lyons 2000; Harrison et al. 2003). After investigating three flare-related EUV Imaging Telescope (EIT) waves, Delannée (2000) found multiple dimmings: a first dimming between the bright front and the flare, a second dimming between the bright front and a magnetic dipole, and a third dimming in transequatorial loops connecting the flare region with another dipole. In this work, the primary dimming surrounding the flare was not obvious. Only remote dimmings appeared directly far from the flare site in the quiet region. The presence of remote dimmings in all EUV wavelengths except $304 \AA$ suggests that the dimmings are interpreted by density depletion rather than temperature variation (Mason et al. 2014). The degrees of intensity decrease are comparable to those of CME-associated dimmings (Dissauer et al. $2018 \mathrm{~b}$ ). The evolution of remote dimmings is characterized by a rapid increase followed by a gradual decay, which is similar to typical CME-associated dimmings (Reinard \& Biesecker 2008). Both the rise and recovery times are close to those of CMEassociated dimmings (Reinard \& Biesecker 2008). The total areas have the same order of magnitude as the CME-associated dimmings and are close to the median value $\left(2.15 \times 10^{4} \mathrm{Mm}^{2}\right)$ (Dissauer et al. 2018b).

After studying two confined flares triggered by the filament eruption, Yang \& Zhang (2018) discovered that the erupting filaments are guided by large-scale field lines and finally reach the remote site (see their Fig. 3). In a follow-up work, Yang et al. (2019) proposed a two-step evolution of a magnetic flux rope, the standard rising phase producing a pair of parallel ribbons and the external reconnecting phase, during which the eruptive flux rope reconnects with the large-scale, overlying CLs (see their Fig. 7). The external reconnection results in transfer and redistribution of the magnetic helicity. Meanwhile, the large-scale loops are heated and filled with hot plasma. A pair of newly formed ribbons are generated in the chromosphere.

In this study, the CRF was also triggered by an eruptive minifilament. During its initial rising phase, the filament pushed the CLs aside and resulted in small-scale, pre-flare dimming, which could be observed only in 131 and $171 \AA$ with formation temperatures of $\log T \approx 5.6-5.8$ in the lower corona. After the onset of CRF, the filament continued to ascend and dilate. However, the confinement from the CLs was so strong that the filament failed to evolve into a CME (Sun et al. 2015). Magnetic reconnection between the EF and CLs may also heat the CLs to high temperature observed in $94 \AA$. Meanwhile, the CLs were continuously being pushed aside and underwent rapid expansion in the southwest direction. Therefore, the large-area remote dimmings, which were associated with the negative footpoints (FP2) of CLs, may result from density depletion of the CLs during their expansion, which is consistent with the fact that large-area dimmings were observed in all EUV passbands except $304 \AA$.

In this work, we investigated the confined M1.1 CRF triggered by the eruption of a mini-filament without a CME on 2015 October 16. A few minutes before the flare HXR peak time, small-scale, weak dimming appeared $\sim 240^{\prime \prime}$ away from the flare. Such pre-flare dimming was observed only in 131 and $171 \AA$. Afterward, long and narrow dimmings became evident in all AIA EUV wavelengths except $304 \AA$, while localized core dimming was absent near the flare site. The large-area dimmings extended southeastward and the areas increased gradually. The total area of remote dimmings reaches $(1.2 \pm 0.4) \times 10^{4} \mathrm{Mm}^{2}$ in $193 \AA$. The maximal relative intensity decreases in 171 and $193 \AA$ reach $90 \%$ and $80 \%$, respectively. Subsequently, the dimmings began to replenish and the area decreased slowly, lasting for $\geq 3 \mathrm{~h}$. The remote dimmings and AR 12434 were connected by large-scale, overlying CLs. The dimmings were associated with the southwest footpoints of CLs with weak negative polarities. We propose that the remote coronal dimmings were probably created by density depletion of the large-scale CLs.

Acknowledgements. The authors are grateful for the referee for valuable suggestions to improve the quality of this article. We thank Y. N. Su, T. H. Zhou, L. Lu, and D. Li in Purple Mountain Observatory for their kind help. SDO is a mission of NASA's Living With a Star Program. AIA and HMI data are courtesy of the NASA/SDO science teams. This work is funded by the NSFC grants (No. 11773079, 11790302, 11603013, U1731101), the Science and Technology Development Fund of Macau (275/2017/A), the International Cooperation and Interchange Program (11961131002), the Youth Innovation Promotion Association CAS, the Strategic Priority Research Program on Space Science, CAS (XDA15052200, XDA15320301), and the project supported by the Specialized Research Fund for State Key Laboratories.

\section{References}

Attrill, G., Nakwacki, M. S., Harra, L. K., et al. 2006, Sol. Phys., 238, 117 Attrill, G. D. R., Harra, L. K., van Driel-Gesztelyi, L., \& Démoulin, P. 2007, ApJ, 656, L101

Attrill, G. D. R., Harra, L. K., van Driel-Gesztelyi, L., \& Wills-Davey, M. J. 2010, Sol. Phys., 264, 119

Cheng, J. X., \& Qiu, J. 2016, ApJ, 825, 37

Delannée, C. 2000, ApJ, 545, 512

Delannée, C., Hochedez, J.-F., \& Aulanier, G. 2007, A\&A, 465, 603

Dissauer, K., Veronig, A. M., Temmer, M., Podladchikova, T., \& Vanninathan, K. 2018a, ApJ, 855, 137

Dissauer, K., Veronig, A. M., Temmer, M., Podladchikova, T., \& Vanninathan, K. 2018b, ApJ, 863, 169

Dissauer, K., Veronig, A. M., Temmer, M., \& Podladchikova, T. 2019, ApJ, 874, 123

Dolla, L. R., \& Zhukov, A. N. 2011, ApJ, 730, 113

Harra, L. K., \& Sterling, A. C. 2001, ApJ, 561, L215

Harra, L. K., Hara, H., Imada, S., et al. 2007, PASJ, 59, S801

Harrison, R. A., \& Lyons, M. 2000, A\&A, 358, 1097

Harrison, R. A., Bryans, P., Simnett, G. M., \& Lyons, M. 2003, A\&A, 400, 1071

Hudson, H. S., Acton, L. W., \& Freeland, S. L. 1996, ApJ, 470, 629

Jin, M., Ding, M. D., Chen, P. F., Fang, C., \& Imada, S. 2009, ApJ, 702, 27

Krista, L. D., \& Reinard, A. A. 2017, ApJ, 839, 50

Lemen, J. R., Title, A. M., Akin, D. J., et al. 2012, Sol. Phys., 275, 17

Lin, R. P., Dennis, B. R., Hurford, G. J., et al. 2002, Sol. Phys., 210, 3

Mandrini, C. H., Pohjolainen, S., Dasso, S., et al. 2005, A\&A, 434, 725

Mandrini, C. H., Nakwacki, M. S., Attrill, G., et al. 2007, Sol. Phys., 244, 25

Mason, J. P., Woods, T. N., Caspi, A., Thompson, B. J., \& Hock, R. A. 2014, ApJ, 789, 61

Mason, J. P., Woods, T. N., Webb, D. F., et al. 2016, ApJ, 830, 20

Miklenic, C., Veronig, A. M., Temmer, M., Möstl, C., \& Biernat, H. K. 2011, Sol. Phys., 273, 125

Qiu, J., \& Cheng, J. 2017, ApJ, 838, L6

Reinard, A. A., \& Biesecker, D. A. 2008, ApJ, 674, 576

Reinard, A. A., \& Biesecker, D. A. 2009, ApJ, 705, 914

Robbrecht, E., \& Wang, Y.-M. 2010, ApJ, 720, L88

Scherrer, P. H., Schou, J., Bush, R. I., et al. 2012, Sol. Phys., 275, 207

Schrijver, C. J., \& De Rosa, M. L. 2003, Sol. Phys., 212, 165

Sun, X., Bobra, M. G., Hoeksema, J. T., et al. 2015, ApJ, 804, L28

Tian, H., McIntosh, S. W., Xia, L., He, J., \& Wang, X. 2012, ApJ, 748, 106

Thompson, B. J., Plunkett, S. P., Gurman, J. B., et al. 1998, Geophys. Res. Lett., 25,2465

Thompson, B. J., Cliver, E. W., Nitta, N., Delannée, C., \& Delaboudinière, J.-P. 2000, Geophys. Res. Lett., 27, 1431

Vanninathan, K., Veronig, A. M., Dissauer, K., \& Temmer, M. 2018, ApJ, 857, 62

Veronig, A. M., Gömöry, P., Dissauer, K., et al. 2019, ApJ, 879, 85

Wang, W., Zhu, C., Qiu, J., et al. 2019, ApJ, 871, 25

Yang, S., \& Zhang, J. 2018, ApJ, 860, L25

Yang, S., Zhang, J., Song, Q., et al. 2019, ApJ, 878, 38

Zarro, D. M., Sterling, A. C., Thompson, B. J., Hudson, H. S., \& Nitta, N. 1999, ApJ, 520, L139

Zhang, Q. M., Li, D., Ning, Z. J., et al. 2016a, ApJ, 827, 27

Zhang, Q. M., Li, D., \& Ning, Z. J. 2016b, ApJ, 832, 65

Zhang, Q. M., Su, Y. N., \& Ji, H. S. 2017, A\&A, 598, A3

Zhang, Q. M., Cheng, J. X., Feng, L., et al. 2019, ApJ, 883, 124

Zhukov, A. N., \& Auchère, F. 2004, A\&A, 427, 705 